\title{
Confidence intervals: a useful statistical tool to estimate effect sizes in the real world
}

\author{
Cecilia Maria Patino, ${ }^{1,2}$ Juliana Carvalho Ferreira ${ }^{2,3}$
}

\section{PRACTICAL SCENARIO}

A prospective cohort study evaluated the association between the presence of asthma and the risk of developing obstructive sleep apnea (OSA) in adults. Adults were randomly recruited from a population-based list of state employees and were followed for four years. Participants with asthma, when compared with those without, had a higher risk of developing OSA in four years (relative risk $[R R]=1.39 ; 95 \% \mathrm{CI}: 1.06-1.82 ; p=0.03$ )

\section{BACKGROUND}

When conducting clinical research, we usually recruit a subgroup of the population of interest in order to increase study efficiency (fewer costs and less time). This subgroup of individuals, the study population, are those individuals who meet the inclusion criteria and agree to participate in the study (Figure 1). We then complete the study and calculate an effect size (e.g., a mean difference or a relative risk) to answer our research question. This process (inference) involves using data collected from the study population to estimate the true effect size in the population of interest, i.e., the source population. In our example, investigators recruited a random sample of state employees (source population) who were eligible and agreed to participate in the study (study population) and reported that asthma increases the risk of developing OSA in the study population ( $R R=1.39)$. To take into account a sampling error due to recruiting only a subgroup of the population of interest, they also calculated a $95 \%$ confidence interval (around the estimate) of 1.06-1.82, indicating a $95 \%$ probability that the true RR in the source population would be between 1.06 and 1.82 .

\section{DEFINITION}

A confidence interval is a measure of imprecision of the true effect size in the population of interest (e.g., difference

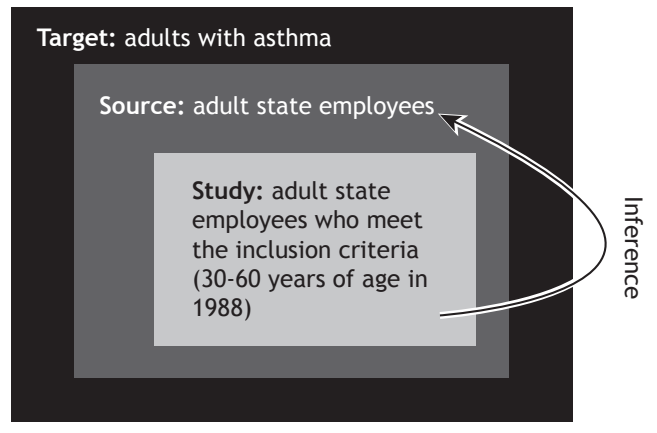

Figure 1. Research populations. between two means or a relative risk) estimated in the study population. That imprecision is due to the sampling error caused by taking subsamples of the population of interest. However, the estimate calculated in the study population is always the best estimate of the effect size in the source population.

\section{WHY DO WE NEED CONFIDENCE INTERVALS?}

We need confidence intervals to indicate the amount of uncertainty or imprecision around the effect size calculated, using the study sample to estimate the true effect size in the source population. Calculating the confidence interval is a strategy that takes into account sampling error: the study effect size and its ' confidence interval represent plausible values for the source population, and the narrower the confidence interval is, the more certain we are that the estimate from the study population represents the true effect size in the source population.

\section{CONFIDENCE INTERVALS: INTERESTING FACTS}

The most common width of confidence intervals reported in the literature is the $95 \%$ confidence interval. However, if we are interested in more or less confidence, $90 \%$ or $99 \%$ confidence intervals can be used.

The confidence interval represents the uncertainty of the effect size in the source population, not in the study population. When calculating a confidence interval, the width of the interval is determined by the sample size (i.e., the individuals who agreed to be studied), the amount of measurement error of the study, and the degree of confidence required.

There is a unique relationship between the $95 \%$ confidence interval and a two-sided $5 \%$ level of significance. When the $95 \%$ confidence interval for differences in effect does not include 0 for absolute measures of association (e.g., mean differences) or 1 for relative measures of association (e.g., odds ratios), it can be inferred that the association is statistically significant ( $p<0.05)$. The advantage of the $95 \%$ confidence interval over the $p$ value is that it provides information about the size of the effect, the uncertainty of the population estimate, and the direction of the effect.

Confidence intervals should always be used in order to describe the major findings of a research study. The relevant confidence intervals should be shown not only in the text of the paper but also in the abstract.

1. Department of Preventive Medicine, Keck School of Medicine, University of Southern California, Los Angeles, CA, USA.

2. Methods in Epidemiologic, Clinical and Operations Research-MECOR-Program, American Thoracic Society/Asociación Latinoamericana de Tórax.

3. Divisão de Pneumologia, Instituto do Coração - InCor - Hospital das Clínicas, Faculdade de Medicina, Universidade de São Paulo, São Paulo, Brasil. 


\section{RECOMMENDED READING}

1. Teodorescu M, Barnet JH, Hagen EW, Palta M, Young TB, Peppard $\mathrm{PE}$. Association between asthma and risk of developing obstructive sleep apnea. JAMA. 2015;313(2):156-64. http://dx.doi.org/10.1001/ jama.2014.17822

2. Sedgwick P. Understanding confidence intervals. BMJ 2014;349:g6051. http://dx.doi.org/10.1136/bmj.g6051
3. Sedgwick P. Confidence intervals: predicting uncertainty. BMJ. 2012;344:e3147. http://dx.doi.org/10.1136/bmj.e3147

4. Ferreira $\mathrm{JC}$, Patino $\mathrm{CM}$. What does the $\mathrm{p}$ value really mean? J Bras Pneumol. 2015;4195):485. doi: 10.1590/S1806-37132015000000215.

5. Gardner MJ, Altman DG. Statistics with confidence: confidence intervals and statistical guidelines. British Medical Journal. Belfast: The Universities Press Ltd; 1989. 